\title{
IMPACT OF WHIP ROLLER PARAMETERS ON WARP DYNAMIC LOADS FOR 3D FABRICS MADE ON A FOUR-COMB WARP-KNITTING MACHINE
}

\author{
Andrzej Michalak ${ }^{1 *}$, Maciej Kuchar², Zbigniew Mikołajczyk \\ 1Department of Knitting Technology, Lodz University of Technology, 116 Żeromskiego Street, 90-924 Łódź, Poland \\ ${ }^{2}$ Department of Vehicles and Fundamentals of machine Design, Lodz University of Technology, 1-15 Stefanowskiego Street, 90-924 Łódź, Poland \\ E-mail: andrzej.stefan.michalak@p.lodz.pl
}

\begin{abstract}
:
The most advantageous geometry of the warp feeding system was determined from the viewpoint of compensating temporary changes in the warp length during the technological cycle of a four-comb warp-knitting machine. Dynamic simulations of the feeding system were carried out for 3 different lengths of the shift of the slider with a guide bar - designating variants (series) of the cross-sectional sizes of the 3D knitted fabrics. The courses of instantaneous warp tensions during the operating cycle of the warp-knitting machine were presented. Limit dynamic loads of the warp were determined and presented as a function of natural frequency of the whip roller. Based on the criterion of the smallest dynamic loads of the warp, the optimum natural frequencies of the whip roller were determined. In the analyzed range of the whip roller parameters, they are 3-6 times greater than the operation frequency of the warp-knitting machine.
\end{abstract}

\section{Keywords:}

Warp tension, whip roller, warp feeding system, natural frequency, stiffness

\section{Introduction}

Spatial textile products frequently constitute preforms for reinforcements in the process of manufacturing composite structural elements for various applications [1, 2]. For the production of knitted fabrics with a 3D structure, knitting machines of specific construction have to be used, e.g., warpknitting machines with several guide bars and needle combs (Fig. 1a) [3].

The four-comb warp-knitting machine which is being considered is characterized by a significant warp demand, due to the nature of the technological process.

It is estimated that warp consumption in case of forming the filling layer of the 3D knitwear is, for full threading, 50 times greater than in case of the side walls, which are formed like a flat knitted fabric, on a classic warp-knitting machine (Fig 1b) $[4,5,6]$. The warp is unwound from the beam with a quasi-
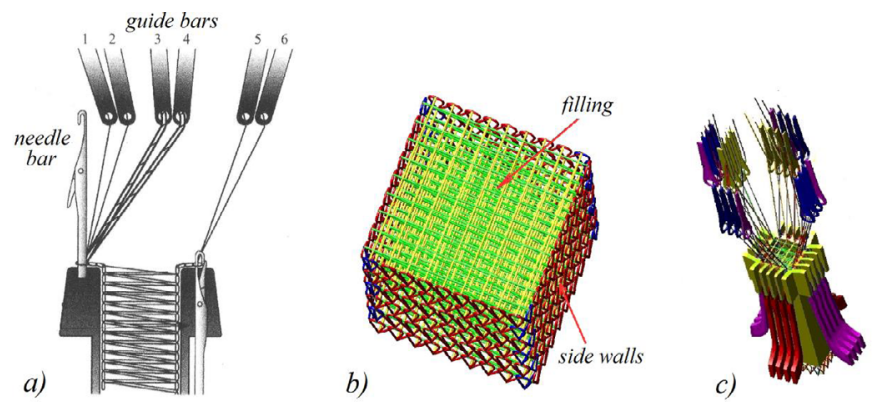

Figure 1. a) Diagram of double-comb warp-knitting machine; b) model of $3 D$ knitted fabric; c) diagram of four-comb warp-knitting machine constant linear velocity (the so-called positive feeding). This velocity corresponds to the average warp demand, over the range of several cycles of machine operation [6, 7]. However, within a single movement cycle of the guide bar, due to its kinematics, the temporary changes in the length of the warp do not fully correspond to the constant expenditure from the beam, and as a result the knitting machine is subjected to considerable dynamic loads [8, 9, 4].

To reduce these loads, the feeding system of the machine is equipped with a relatively long whip roller.

Its task is to compensate the temporary changes in the warp length. The whip roller operates in a passive manner and is characterized by rotational stiffness [9, 10, 11]. Increasing the length of the whip roller not only rises its compensation capabilities but also results in the increase in the moment of inertia, which in turn reduces its natural frequency and thus increases the negative impact of whip roller dynamics on temporary warp tension. As a consequence, there is a need to increase either the stiffness of the whip roller or the pretension of the warp [12], which obviously negatively influences the warp loads. Therefore, an important problem is the optimization of the whip roller parameters toward reduction in warp loads during the knitting process

\section{Model of warp feeding system}

A diagram of the warp feeding system of a four-comb warpknitting machine is shown in Fig $2 b$. The main elements of the feeding system are as follows: beam 1, articulately supported 


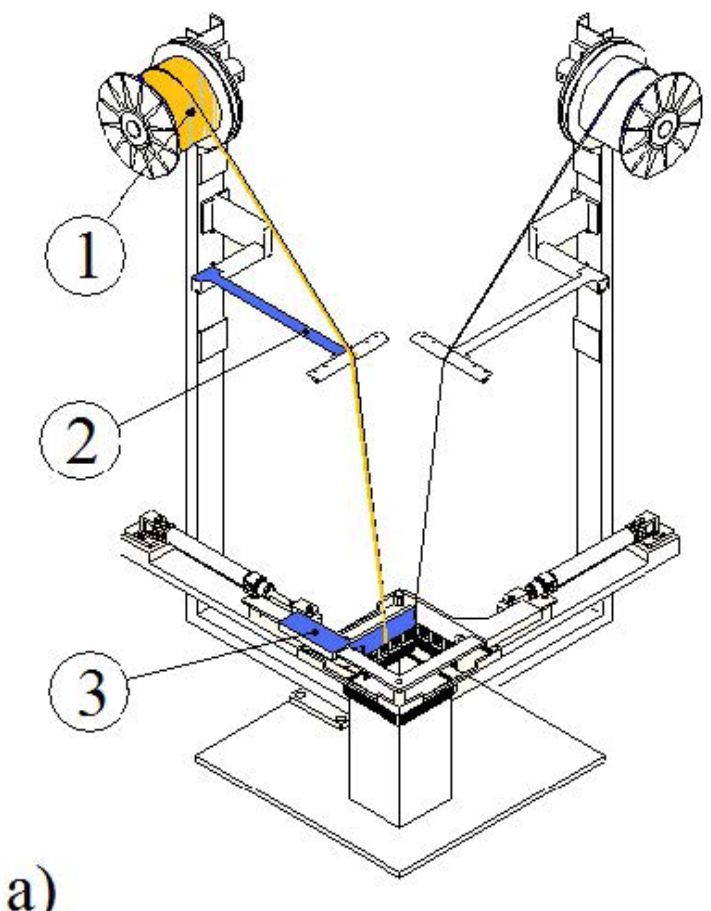

a)

Figure 2. Geometry of warp feeding system: a) model; b) diagram.

whip roller 2 with length $r$ and the slider system of the guide bar 3. The slider performs reciprocating horizontal motion, with technological shutdowns. The beam, the whip roller, and the slider are combined by the warp with length L. Warp expenditure during the operation of the warp-knitting machine results from thread demand in the knitting zone, i.e., kinematics of the loop-forming elements: horizontal motion of the slider $s$ and, during the slider shutdowns, vertical motion of the needle combs $u(u=10 \mathrm{~mm})[5,7]$. The momentary demand is only partly covered by the warp section w unwound from the beam. The missing part of the warp length $\Delta \mathrm{L}$ is compensated by the section resulting from the deflection of the whip roller. This length results from the relationship (1), and the components of which are shown in Fig. 3.

$$
\Delta L=s-(w-u)
$$

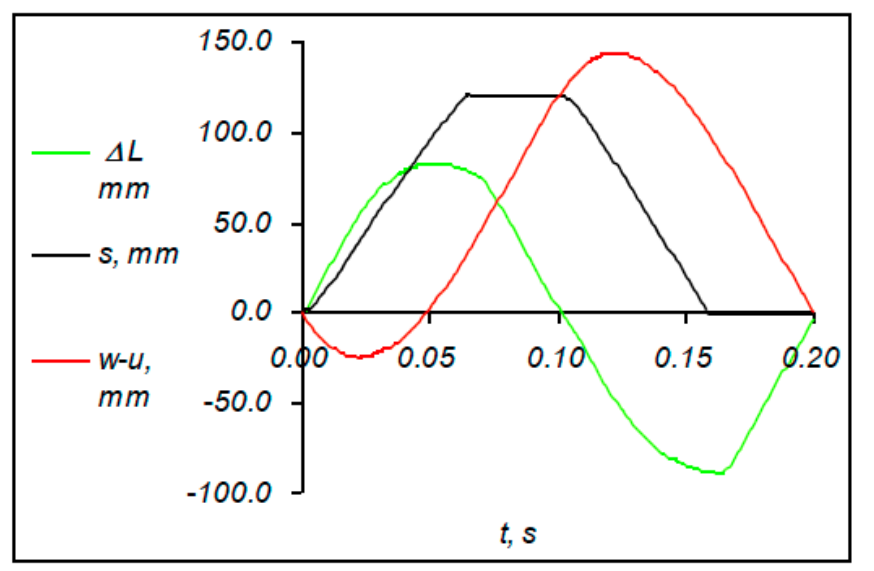

Figure 3. Changes of warp length during the cycle and its components (with slider stroke $S=120 \mathrm{~mm}$ )

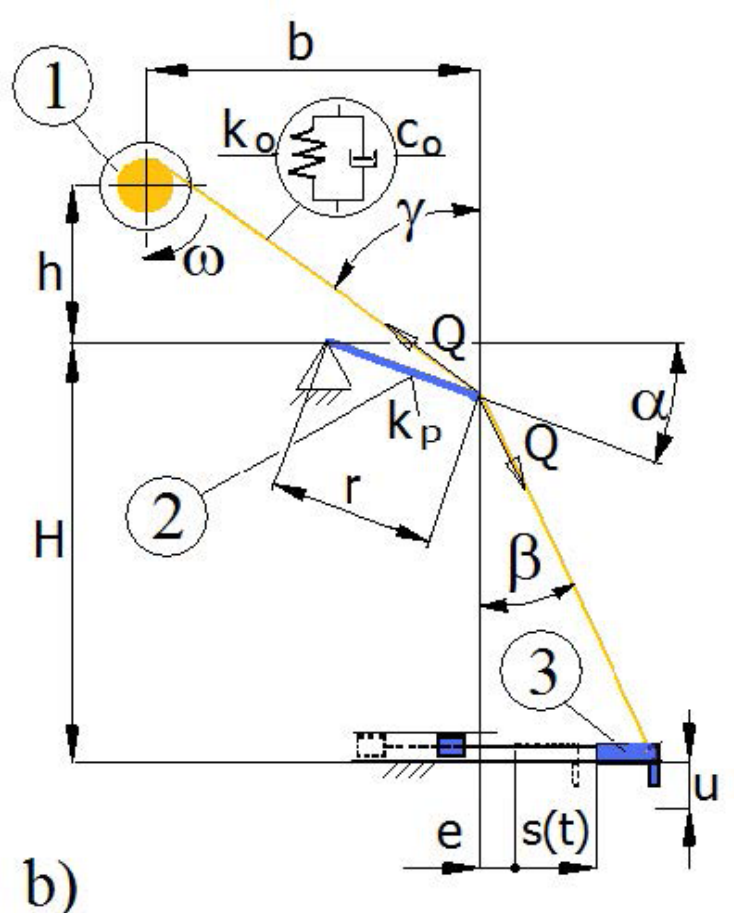

At first, the geometry of the feeding system during motion was analyzed assuming that the warp is inextensible and the articulately supported whip roller is weightless. Due to the length compensating function $\Delta \mathrm{L}$, the required limit value am of the rotation angle of the whip roller a must satisfy the dependence (2). While maintaining constant length of the warp $L$, equal to its initial value, the value am corresponds approximately to full slider shift $\mathrm{S}$.

$L=\sqrt{\left(h+r \sin \alpha_{m}\right)^{2}+\left(b-r\left(1-\cos \alpha_{m}\right)\right)^{2}}+\sqrt{\left(H-r \sin \alpha_{m}\right)^{2}+\left(S+e+r\left(1-\cos \alpha_{m}\right)\right)^{2}}-u$

where $e$ is the location of support axis of the whip roller with respect to the starting point of slider path measured in horizontal direction toward slider movement axis.

On the other hand, the angle $\alpha$ is limited by the geometry of the feeding system, from which its permissible value $\alpha_{d}$ results, determined as dependent on the limiting angles $\alpha_{a}$ and $\alpha_{b}$. The whip roller cannot turn by an angle larger than $\alpha_{a}$, for which the warp section between the beam and the whip roller would lean against the axis of the whip roller, according to the dependence (3):

$$
\alpha_{a}=\operatorname{arctg} \frac{h}{b-r}
$$

The momentary rotation angle of the whip roller cannot also reach value $\alpha_{b}$. If that happened, the whip roller would break away from the warp, stretched directly between the beam and the slider. This angle is related to geometrical parameters: $H$, $S, r$, according to dependence (4).

$$
\frac{H-r \sin \alpha_{b}}{e+S+r\left(1-\cos \alpha_{b}\right)}=\frac{h+H}{b+S+e}
$$

The greatest permissible value of the rotation angle of the whip roller $\alpha_{d}$ is determined by the lower angle values $\alpha_{a}$ and $\alpha_{b}$. 
The ranges of geometric values of the feeding system adopted in the analysis are as follows: $H=440-1300 \mathrm{~mm}, h=100$ $500 \mathrm{~mm}, r=24-264 \mathrm{~mm}, b=300 \mathrm{~mm}, e=10 \mathrm{~mm}, u=10 \mathrm{~mm}$.

Taking into account the actual dynamic operation of the whip roller and the warp, the equation of rotational motion of the whip roller was introduced, according to the relationship (5 and $6)$ :

$$
\begin{aligned}
& Q \cos (\beta+\alpha) r-Q \cos (\delta+\alpha) r=k_{p} \alpha+J_{p} \frac{d \alpha}{d t} \\
& \operatorname{tg} \beta=\frac{r(1-\cos \alpha)+e+s}{H} ; \operatorname{tg} \delta=\frac{b-r(1-\cos \alpha)}{h+r \sin \alpha}
\end{aligned}
$$

Momentary tension force of the warp $Q$ was determined according to the dependence (7):

$$
Q=Q_{0}+\Delta L k_{o}+c_{o} \frac{d L}{d t}
$$

The whip roller, with length $r=70-264 \mathrm{~mm}$ (weight in the range: $m_{p}=0.031-0.125 \mathrm{~kg}$, moment of inertia in the range: $J_{p}=100-3614 \times 10-6 \mathrm{~kg} \times \mathrm{m}^{2}$ ), rotates by angle $\alpha$ in relation to the axis with rotational rigidity in the range $k_{p}=0-2000 \mathrm{~N} / \mathrm{mm} /$ deg. It has been assumed that the damping on the axis of the whip roller and frictional coefficient of the warp against the whip roller beam are negligible [13, 14, 15]. The warp with length $L$, possessing elastic-plastic properties: stiffness $k_{o}=20 \mathrm{~N} /$ $\mathrm{mm}$ and viscous damping coefficient $c_{0}=0.01 \mathrm{~N} / \mathrm{mm}$, during the mechanism operation is strained with force $Q$ [16-20]. The initial tension of the warp $Q_{0}$ results from the need to ensure the appropriate technological level of the process [6, 8]. It was assumed that it equals $50 \mathrm{~N}$ in total for all the threads on the given guide bar.

\section{Geometric constraints resulting from shifts of the slider and the whip roller}

In dynamic conditions, the limit values of the rotation angle of the whip roller a may differ from the required values $\alpha_{m}$, and in special cases they may be greater. However, they should not exceed the permissible values $\alpha_{d}$. To leave as much space as possible for dynamic phenomena, the geometry of the feeding mechanism of the warp-knitting machine should guarantee

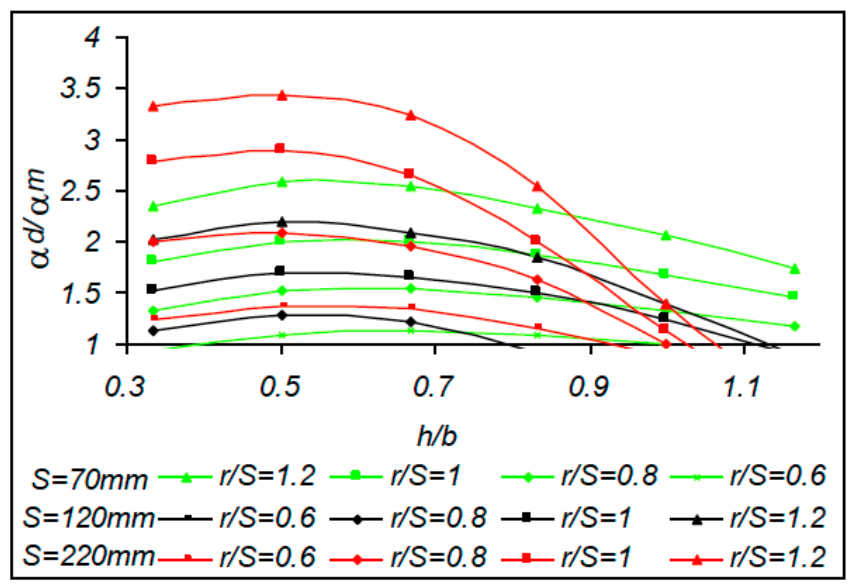

Figure 4. Numeric values of the $\alpha_{d} / \alpha_{m}$ ratio resulting from the relation of the permissible to the required rotation angle of the whip roller, as a function of relative height of beam axis position the highest possible value of the $\alpha_{d} / \alpha_{m}$ ratio, adopted in this analysis.

Based on the dependencies (2-4), the permissible and required rotation angles of the whip roller were determined. Fig. 4 shows the values of the $\alpha_{d} / \alpha_{m}$ ratio as a function of relative height of the beam axis, measured from the axis of whip roller $h / b$. The individual points of the presented curves were obtained for different relative lengths of the whip roller, related to the slider shift length $r / S$. The results for 3 different values of the slider shift were superimposed. The largest numerical values of the $\alpha_{d} / \alpha_{m}$ ratio were obtained for height $h / b=0.5-0.7$ and for the length of the whip roller $r / S=1-1.2$.

Fig. 5 shows the values of the $\alpha_{d} / \alpha_{m}$ ratio as a function of relative height of the rotation axis of the whip roller, measured from the slider motion level and related to the slider shift $H / S$. The individual points of the curves were obtained for different relative lengths of the whip roller $r / S$. The results for 3 lengths of the slider shift $S$ were superimposed. The highest values of the $a_{d} / a_{m}$ ratio were obtained for the height of the rotation axis of the whip roller $H / S=5-7$, depending on the length of the slider shift $S$ and the length of the whip roller $r / S=1-1.2$. For further considerations, the following geometric assumptions were adopted for the feeding mechanism: $h / b=0.6 \mathrm{H} / \mathrm{S}=4-7$, $r / S=1-1.2$.

\section{Simulation of dynamic tests of the warp feeding system}

During the actual operation of the warp feeding mechanism, the warp is subjected to temporary deformations (elongations) and the whip roller operates dynamically. Equations (5) and (7) were solved by dynamic simulations for the assumed time course of the slider motion (Fig. 3). Fig. 6 shows some examples of instantaneous values of warp tension $Q$ during a single knitting cycle for different rotational stiffnesses of the whip roller $k_{p}$.

Based on the obtained simulation results, the warp tension was analyzed in terms of its limit values. Generally, the peak values

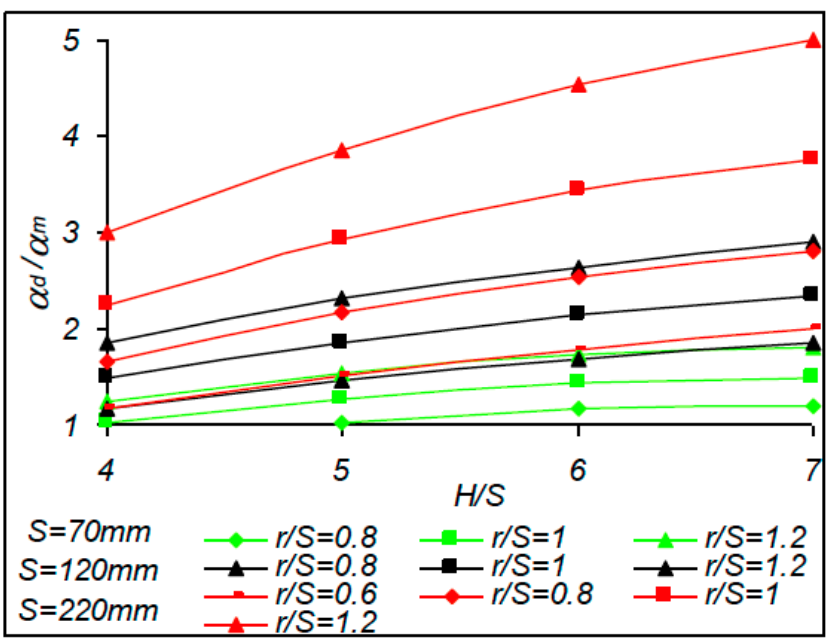

Figure 5. Numerical values of the $\alpha_{d} / \alpha_{m}$ ratio as a function of the relative height of the rotation axis position of the whip roller 


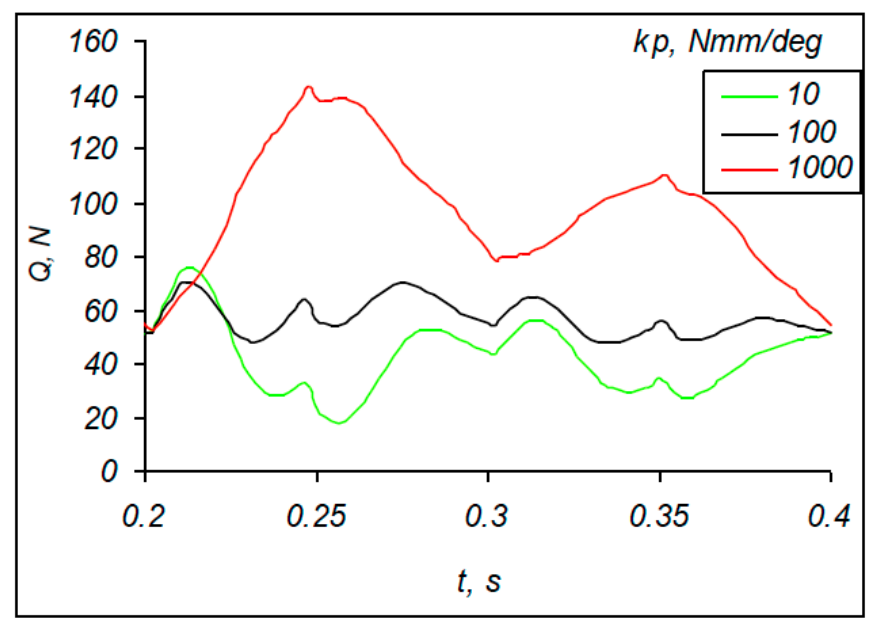

Figure 6. Instantaneous force values in the warp during single knitting cycle, $S=120 \mathrm{~mm}$

of the warp tension force $Q_{\max }$ are high for higher stiffness of the whip roller. With the reduction in the stiffness of the whip roller, the tension in the warp also decreases, and the whip roller better fulfills its compensating function. However, together with excessive reduction in the stiffness of the whip roller, the peak values of warp tension increase again. Temporary reduction in the minimum value of warp tension $Q_{\min }$ is also visible, to the level below the technologically necessary value. As a result, the initial tension of the warp has to be increased. The analysis of the courses of warp tension allowed to identify the range of optimum whip roller parameters, defined by its stiffness and moment of inertia.

To preserve the general nature of the considerations, the parameters, such as rotational stiffness and the moment of inertia of the whip roller, were linked together as natural frequency of the whip roller $f_{p}$.

Fig. 7 presents the limit values of the warp tensions occurring during the knitting cycle as a function of the natural frequency of the whip roller. The results are presented in relative form. The peak warp tensions were related to the level of necessary technological tension $Q_{0}$. The natural frequency of the whip roller was referred to the frequency of the warp-knitting machine $f_{c}$. The results obtained for 3 different shift lengths of the slider are presented. During the simulations, when the slider shift was extended, the obtained frequencies of the warp-knitting machine diminished: $f_{c}=6.25 \mathrm{~Hz}$ for shift $S=70 \mathrm{~mm}, f_{c}=5 \mathrm{~Hz}$ for shift $S=120 \mathrm{~mm}$ and $f_{c}=3.8 \mathrm{~Hz}$ for shift $S=220 \mathrm{~mm}$ [9]. The range of the analyzed natural frequencies of the whip roller was $f_{p}=10-90 \mathrm{~Hz}$.

Fig. 8 presents the peak angles $\alpha_{\max }$ of the whip roller rotation as a function of natural frequency of the whip roller. The results are presented in relative form. Peak values of the angles were referred to the required angle from the initial considerations $\alpha_{m a x} / \alpha_{m}$.

\section{CONCLUSIONS}

The results of static preliminary analysis made it possible to identify the geometric constraints for the feeding mechanism

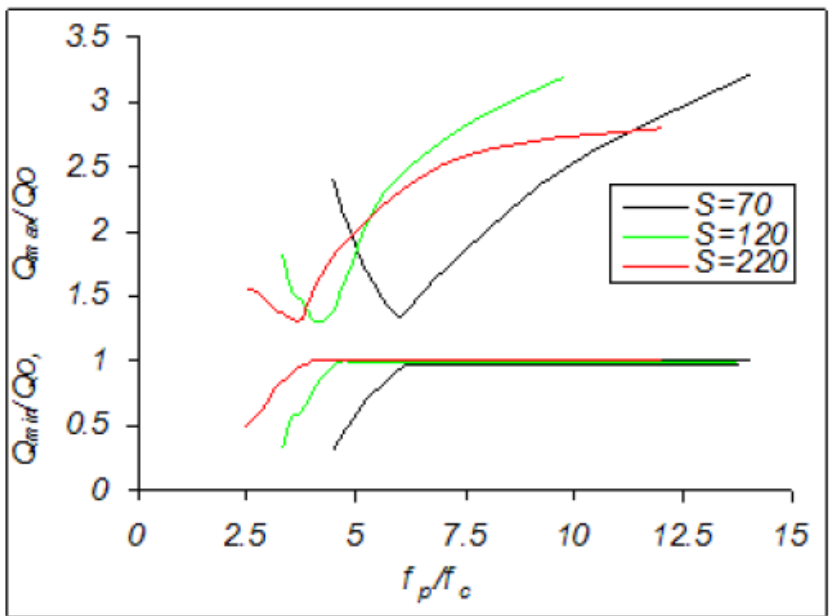

Figure 7. Limit values of dynamic warp loads, related to technological tension, as a function of the relative natural frequency of the whip roller

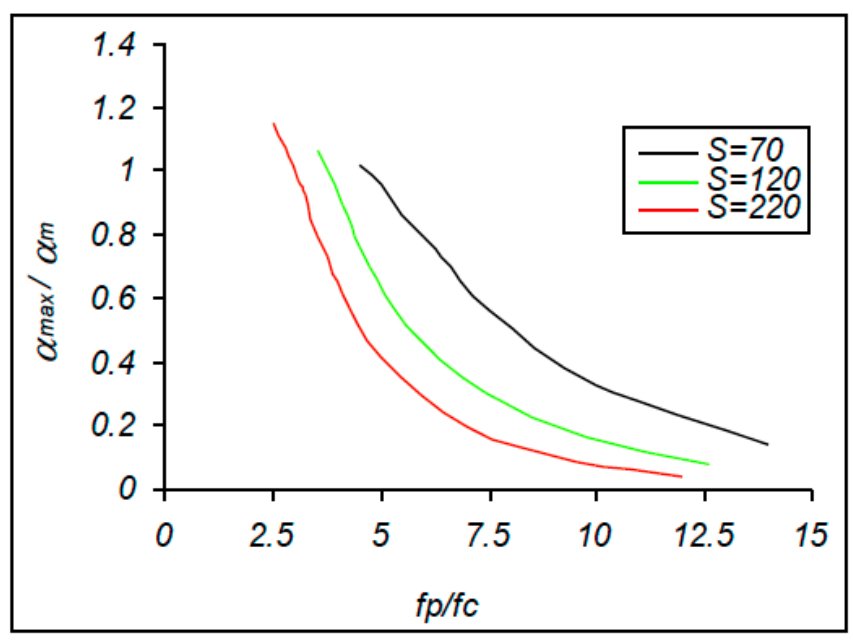

Figure 8. Limiting rotation angle of the whip roller, related to its required value, as a function of relative natural frequency of the whip roller.

of the warp-knitting machine $(h / b=0.6 \mathrm{H} / \mathrm{S}=4-7, \mathrm{r} / \mathrm{S}=$ 1-1.2). The obtained data constitute important constructional assumptions.

The performed dynamic simulations of the feeding system of the warp-knitting machine indicate the necessity for optimizing the parameters of the key element which is the whip roller. To meet the criterion of the smallest possible dynamical loads on the warp, whose level in the model tests was about $140 \%$ of the required technological force $\left(Q=1.4 Q_{0}\right)$ - the geometric and material parameters of the whip roller should shape its natural frequency as several times higher than the frequency of the warp-knitting machine, in the range $f_{p} / f_{c}=3-6$. Beyond this frequency range, the peak warp loads increase, and then the excessive temporary decrease in the warp tension below its necessary technological level is also observed. On the basis of the course of the warp tension, the optimum parameters of the whip roller, such as rotational stiffness and moment of inertia, were determined.

The length of the slider shift (related to the technological size of the product) affects the limit loads on the warp in a limited manner. For the shortest analyzed shift $S=70 \mathrm{~mm}$, the optimum value of the $f_{p} / f_{c}$ ratio is higher and amounts to 6 . For 
the longest shift of the slider $S=220 \mathrm{~mm}$, the optimum value of the $f_{p} / f_{c}$ ratio is smaller and amounts to 3 .

The results of dynamic simulations indicate that in the range of optimum values of the natural frequency of the whip roller $\left(f_{p} / f_{c}=3-6\right)$, the maximum rotation angle of the whip roller $\alpha_{\max }$ does not exceed its required values $\alpha_{m}$. Thus, the danger of exceeding the permissible values $\alpha_{d}$ is even less probable.

\section{References}

[1] Radaszewska, E., Benda, A., Korycki, R. (2018). Modelling of textile composite adapted for roof structure. Fibres \& Textiles in Eastern Europe, 26, 5(131), 99-103, DOI: 10.5604/01.3001.0012.2539.

[2] Landesmann, A., Seruti, C. A., Batista, E. (2015). Mechanical properties of glass fiber reinforced polymers members for structural applications. Materials Research. 18(6), 1372-1383, DOI: tttp://dx.doi.org/10.1590/15161439.044615.

[3] Xiaogang, C. (2015). Advances in 3D Textiles. Woodhead publishing is an imprint of Elsevier 2015, Series in Textiles Number 167.

[4] Mikołajczyk, Z. (2009). Modeling of the knitting process with respect to the optimisation of the construction parameters of warp-knitting machines. Fibres \& Textiles in Eastern Europe, 17, 2 (73), 76-81.

[5] Pieklak, K., Mikołajczyk, Z. (2009). Original concept of a new multicomb warp-knitting machine for manufacturing spatial knitted fabrics. Fibres \& Textiles in Eastern Europe, 17, 3 (74), 76-80.

[6] Michalak, A., Kuchar, M., Mikołajczyk, Z. (2015). Simulation tests of the feeding system dynamics on the warp knitting machine with four needle combs. Fibres \& Textiles in Eastern Europe, 23, 4 (112), 127-133.

[7] Michalak, A., Kuchar, M., Mikołajczyk, Z. (2017). Dynamic analysis of a warp-knitting machine with pneumatic drive for producing 3D knitted fabrics. Indian Journal of Fibre \& Textile Research, 42, December, 502-505.

[8] Mikołajczyk, Z. (2011). Optimisation of the knitting process on warp-knitting machines in the aspect of the feeding zone geometry. Fibres \& Textiles in Eastern Europe, 19, 4 (87), 81-88.
[9] Čiukas, R., Sadauskas, D. (2008). Control of the stability circular knitting process with passive yarn feeding. Mechanika, 4(72), 77-81.

[10] Çelik, Ö., Eren, R. (2014). Mathematical analysis of warp elongation in weaving machines with positive backrest system. Tekstil ve Konfeksiyon, 24(1), 56-64.

[11] Mikołajczyk, Z. (2003). Model of the feeding process of anisotropic warp knitted fabrics. Fibres \& Textiles in Eastern Europe, 11, 2(41), 58-62.

[12] Mikołajczyk, Z. (2011). Optimisation of the knitting process on warp-knitting machines in the aspect of the properties of modified threads and the vibration frequency of the feeding system. Fibres \& Textiles in Eastern Europe, 19, 6 (89), 75-79.

[13] Pusch, T., Wünsch, I., Offermann, P. (2000). Dynamics of yarn tension on knitting machines. Autex Research Journal, 1, 2, 55-63.

[14] Bedez Ute, T. (2016). A comparative study between the dynamic friction characteristics of compact and siro yarns. Tekstil ve Konfeksiyon, 26(1), 55-63.

[15] Svetnickienè, V., Čiukas, R. (2009) Investigation of friction properties of yarns from natural fibres. Mechanika, 1(75), 73-77.

[16] Zajączkowski, J. (2017). Vibrations of elastically supported masses separated by a textile layer. Fibres \& Textiles in Eastern Europe, 25, 1(121), 131-133, DOI: 10.5604/12303666.1227894.

[17] Shinde, D., Kimbro, E., Mohan, R., Kelkar, A. (2013). Mechanical properties of woven fiberglass composite interleaved with glass nanofibers. The 19th International Conference on Composite Materials, Montreal, Canada.

[18] Kuchar, M. (2016). The impact of the frequency of reed vibrations on improving the conditions in thickening dense technical fabrics. Tekstil ve Konfeksiyon, 26(4), 380-384.

[19] Ou, J., Zhu, D., Zhang, H., Huang, I., Yao, Y., Li, G. (2016). Mechanical characterization of the tensile properties of glass fiber and its reinforced polymer (GFRP) composite under varying strain rates and temperatures. Polymers, 8, 196-212, DOI: 10.3390/polym8050196.

[20] Iqbal, S., Eldeeb, M., Ahmad, Z., Mazari, A. (2017). Comparative study on viscose yarn and knitted fabric made from open end and rieter airjet spinning system. Tekstil ve Konfeksiyon, 27(3), 234-240. http://dergipark. gov.tr/tekstilvekonfeksiyon/issue/31290/340940. 